\title{
Sujetos de la Reforma: Condiciones institucionales para la implementación de la RS
}

\author{
Carmen Griselda Loya Ortega \\ Centro Chihuahuense de Estudios de Posgrado \\ grisyloya@cchep.edu.mx
}

\begin{abstract}
Resumen
Para efectos del presente artículo se toma como unidad de análisis las características de los sujetos que encabezan las escuelas de nivel secundaria (directores y subdirectores) y una breve reseña respecto de las condiciones institucionales que existen para implementar la reforma en este nivel en la ciudad de Chihuahua, específicamente la caracterización del personal que integra las escuelas de este nivel. La información que se presenta forma parte de una investigación más amplia cuyo propósito fue indagar sobre la reforma y los cambios que se generan durante su implantación en las percepciones, intereses y expectativas de quienes encabezan las escuelas secundarias. El estudio se planteó como un seguimiento en tres momentos durante los ciclos escolares 2006-2007 y 20072008; se trata de una investigación enmarcada en el paradigma cualitativo bajo el enfoque del interpretacionismo simbólico con la inclusión de elementos cuantitativos que nos permiten caracterizar ampliamente a los sujetos participantes. Sobre esa base se intenta develar los significados que los individuos construyen en función de sus interacciones con los sujetos que integran las instituciones y el propio fenómeno de la reforma. Se analiza el proceso que sigue la perspectiva de los directivos en los tres momentos del estudio y se obtienen resultados que reflejan avance en el conocimiento de algunos de los factores institucionales que se ven inmersos en el proceso de implantación de la reforma.
\end{abstract}

\section{Palabras clave}

Reforma educativa, perspectivas, directores.

\section{Introducción}

Con base en el seguimiento efectuado en los primeros años en los que da inicio la reforma en el nivel de secundaria se posibilitó el rescate de la perspectiva de los directivos respecto de los elementos institucionales que posibilitan u obstaculizan la incorporación de acciones que influyen en la dinámica de institucionalización de la RS.

En el estudio se le atribuye trascendencia al rol que desempeñan los responsables de los centros escolares en la promoción de los cambios al afrontar los retos que supone la implantación de un nuevo currículo. La presentación de los resultados se centra en la descripción de las características del personal directivo que participa en la investigación.

La visión de los sujetos que encabezan las escuelas secundarias públicas del subsistema estatal en la Ciudad de Chihuahua se analiza a partir de dos dimensiones: la pedagógica y la organizacional; en ellas se ubican los hallazgos obtenidos a lo largo de las 
tres etapas del seguimiento. Para efectos del presente trabajo se incluye información que permite caracterizar tanto a los sujetos como las condiciones institucionales en las que se implanta la RS.

Como parte de los resultados y en atención al enfoque metodológico del estudio, se incluye una interpretación, considerando para ello la incidencia de factores que favorecen o limitan el logro de los objetivos planteados para la reforma.

\section{La función directiva}

Existe una idea difundida entre los integrantes del sistema educativo en la que se otorga un carácter instrumental al papel del directivo, se considera como un agente que "se concentra en atender asuntos administrativos, en los trámites para el mantenimiento y mejoramiento del edificio escolar y (...) en demandas adicionales de la institución como la organización de eventos culturales, sociales y deportivos" (SEP, 2004, p. 12). Esta visión reduccionista presumiblemente margina otras implicaciones que tiene la función directiva; como figura administrativa es el líder formal de la institución y su desempeño se determina por un Reglamento Interior de Trabajo de las Escuelas, sus responsabilidades son hasta cierto punto confusas pues "al directivo le corresponde resolver todos los problemas que tengan que ver directa o indirectamente con la escuela" (Elizondo, 2001, p. 34).

Los directivos no son los actores a los que se les confiere directamente la puesta en marcha de la reforma, pues no se encuentran en contacto inmediato con los alumnos; sin embargo, por la naturaleza de sus funciones, les corresponde coordinar y apoyar al equipo de docentes, en quienes recae de manera explícita la implementación pedagógica de la reforma. Por ello, resulta interesante conocer cómo visualizan el progreso, la evolución y los ajustes que genera la implantación de la reforma en la institución que dirigen.

La Reforma a la Educación Secundaria plantea nuevos retos a quienes les están asignadas las funciones directivas en una escuela de este nivel, debido a que representa una oportunidad para modificar, cambiar e incorporar estrategias que influyen en la dinámica de las instituciones. Se asume que los cambios que se deben gestar a partir de la reforma se relacionan directamente con el papel que desempeñan las personas que tienen a su cargo las funciones directivas en las escuelas. Es por ello que surgió el interés en conocer las implicaciones que se presentan con la RS considerando el análisis del fenómeno desde la perspectiva de los directivos y los cambios que se generan durante el proceso de su implantación en las actitudes, las percepciones, intereses, $y$ expectativas de quienes encabezan las escuelas secundarias; contrastándolo con lo planteado como aspiración en los documentos que dan origen a esta reforma.

A grandes rasgos, a través de la Reforma que se emprende en el 2006 se pretende incrementar la calidad de las experiencias de los estudiantes que cursan la educación secundaria, y articular pedagógicamente este nivel con los de educación preescolar y primaria (SEP. 2006).

El logro de esos fines se encuentra supeditado en gran medida a la organización que se dé al interior de los centros educativos, así como la capacidad para coordinar y orientar las acciones hacia la consecución de lo planteado en la RS; en ello, resulta imprescindible el papel de quienes cotidianamente tienen a su cargo las funciones directivas de una institución educativa a fin de que su intervención se encamine en el sentido de mejorar la eficiencia y maximizar las potencialidades de la escuela para alcanzar los objetivos y metas planteadas para la educación básica. Con base en estas 
consideraciones se plantea el propósito y los cuestionamientos que guían el desarrollo de la investigación.

\section{Propósito}

Conocer las perspectivas de los directores de escuelas secundarias de la Cd. de Chihuahua respecto de la implementación de la Reforma a la Educación Secundaria, durante los ciclos escolares 2006-2007 y 2007-2008.

\section{Pregunta central de investigación}

Con base en el propósito de la investigación se plantea el problema de la siguiente manera: ¿Cómo perciben los directivos de secundaria la implementación de la reforma?

\section{Preguntas de investigación}

Algunos de los cuestionamientos que guían la investigación son los siguientes:

- ¿Cuáles son las percepciones que tienen quienes ejercen las funciones directivas respecto de la RS?

- ¿Cuáles son las características de los sujetos que encabezan las instituciones de nivel de secundaria y cómo coadyuvan o interfieren en los procesos de implantación de la RS?

\section{Consideraciones Metodológicas}

La investigación brinda una interpretación de los puntos de vista sobre la implementación de la reforma de quienes encabezan las escuelas secundarias, dado que esto se asume como un fenómeno complejo y de carácter subjetivo se enmarca dentro del paradigma cualitativo. Se emplea el método del interpretacionismo simbólico, pues aspira al conocimiento de la realidad a través de la develación de los significados que los individuos construyen en función de sus interacciones con los objetos, sujetos y fenómenos con los que entran en contacto.

Se considera importante para este estudio hacer visible la perspectiva de los directivos en distintos momentos, a raíz de que los significados construidos por los individuos no resultan permanentes, éstos se modifican con las interacciones que establecen con otros seres y con el mismo fenómeno.

En función de los recursos disponibles se determina que, aun y cuando se aspira a interpretar la perspectiva de los directivos, se pretende ir más allá de la realidad individual; por ello, se rescata la visión global de los participantes a partir de la aplicación de cuestionarios en las tres etapas del seguimiento durante los ciclos escolares 2006-2007 y 2007-2008, complementándola a partir de la información recabada con entrevistas individuales semiestructuradas.

\section{Resultados}

Con base en los resultados del estudio se incluyen elementos que permiten caracterizar a los sujetos que encabezan las instituciones del nivel de secundaria en la ciudad de Chihuahua. Contiene también datos de las condiciones institucionales en las que se implanta la reforma.

¿Quiénes dirigen las escuelas de nivel secundaria?

El estudio se llevó a cabo con directivos de las 26 escuelas estatales públicas ubicadas en la ciudad de Chihuahua, Chih. Como se ha mencionado, para efectos de este estudio se considera como directivos tanto a los directores, como a los subdirectores de los centros escolares del nivel de secundaria.

La cantidad de directivos participantes varía en cada una de las etapas, esto se puede apreciar en la Tabla 1.

Tabla 1. Sujetos participantes por etapa

\begin{tabular}{cccc}
\hline Etapa & Director & Subdirector & Total \\
\hline $\mathbf{1}$ & 24 & 22 & 46 \\
$\mathbf{2}$ & 22 & 18 & 40 \\
$\mathbf{3}$ & 19 & 14 & 33 \\
\hline
\end{tabular}


La edad de los directivos oscila entre los $35 \mathrm{y}$ los 65 años y las frecuencias de las edades se distribuye como lo muestra la Figura 1 que a continuación se presenta.

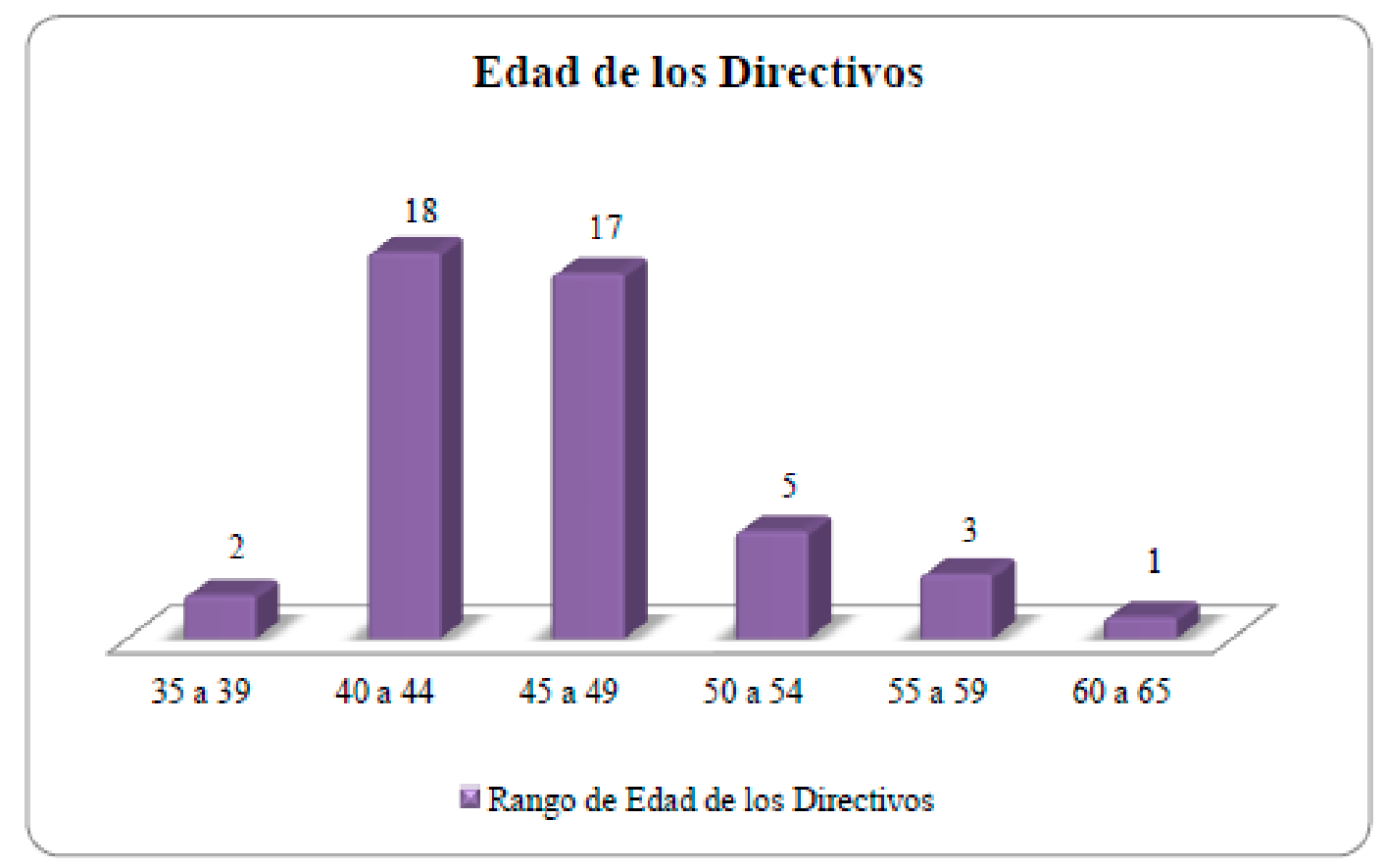

Figura 1. Edad de los directivos.

Cabe destacar que los dos sujetos que muestra la Figura 1, ubicados en el rango de edad entre 35 y 39 años son subdirectores; es decir, todos los sujetos que ocupan un puesto de director son mayores de 40 años de edad. Sólo cuatro directores son mayores de 55 años. Por tanto, tres cuartas partes de los directivos tienen edades comprendidas entre los 40 y 49 años.

Las circunstancias que puede generar esta situación guardan relación con el impacto en el proceso de consolidación de la reforma en el nivel de secundaria, ya que cerca de la mitad de los sujetos que encabezan las instituciones de este nivel permanecerán en el sistema educativo por lo menos durante 12 años, dadas las condiciones actuales del régimen de jubilación en el subsistema estatal. En él, las mujeres se jubilan a los 28 años de servicio y los hombres a los 30; por lo tanto, las edades que tienen los directivos conjugan la experiencia y el futuro del desempeño; ambos constituyen una fortaleza que permite dirigir y seguir el proceso de la reforma hasta su consolidación. 




Figura 2. Tipo de nombramiento.

Del total de directivos, 33 ostentan una plaza dictaminada, 5 tienen el nombramiento de interinos y 7 son encargados de la dirección de la escuela en la que laboran, esta información se encuentra en la Figura 2. Los interinos y encargados en puestos directivos representan cerca de la tercera parte de los directivos con puestos dictaminados, ello implica para los centros, tener que laborar bajo sistemas de organización inestables que ineludiblemente repercutirán en los procesos de gestión que se generan en las instituciones.

El 76\% de los directivos accedió al puesto por concurso escalafonario y sólo 10 de 46 maestros fueron asignados en forma directa para ocupar el puesto. Esto proporciona información respecto del fortalecimiento de los procesos de elección de personal que dirigirá las instituciones educativas en el nivel. El sistema de puntajes para acceder a un puesto directivo, así como el proceso riguroso para dar cuenta de las puntuaciones, asegura de alguna manera que quienes sean asignados para liderar los centros, se hayan sometido a procesos de profesionalización, dejando a un lado la práctica que permitía el acceso a quienes tenían los contactos adecuados para otorgarle un puesto de confianza, o aquellos que sumaban más años de servicio en el sistema educativo.

Los puestos directivos de las escuelas secundarias estatales de la ciudad de Chihuahua los ocupan en su mayoría personas que pertenecen al género masculino. De acuerdo con datos rescatados durante la tercera etapa, se encontró que de los 14 puestos de subdirección sólo cuatro son ocupados por mujeres, en los puestos de dirección se presenta la misma tendencia. Por tanto, como se muestra en la Figura 3, sólo nueve de los 33 directivos pertenecen al género femenino.

Lo anterior no encuentra correspondencia con la proporción de docentes del género femenino que labora en las escuelas del subsistema estatal en la ciudad de Chihuahua. Se obtuvo información que confirma la afirmación con la que inicia el párrafo al solicitar informes respecto del género de los docentes; estas referencias forman parte de los datos contextuales que los directivos proporcionaron durante la primera etapa de la investigación. 


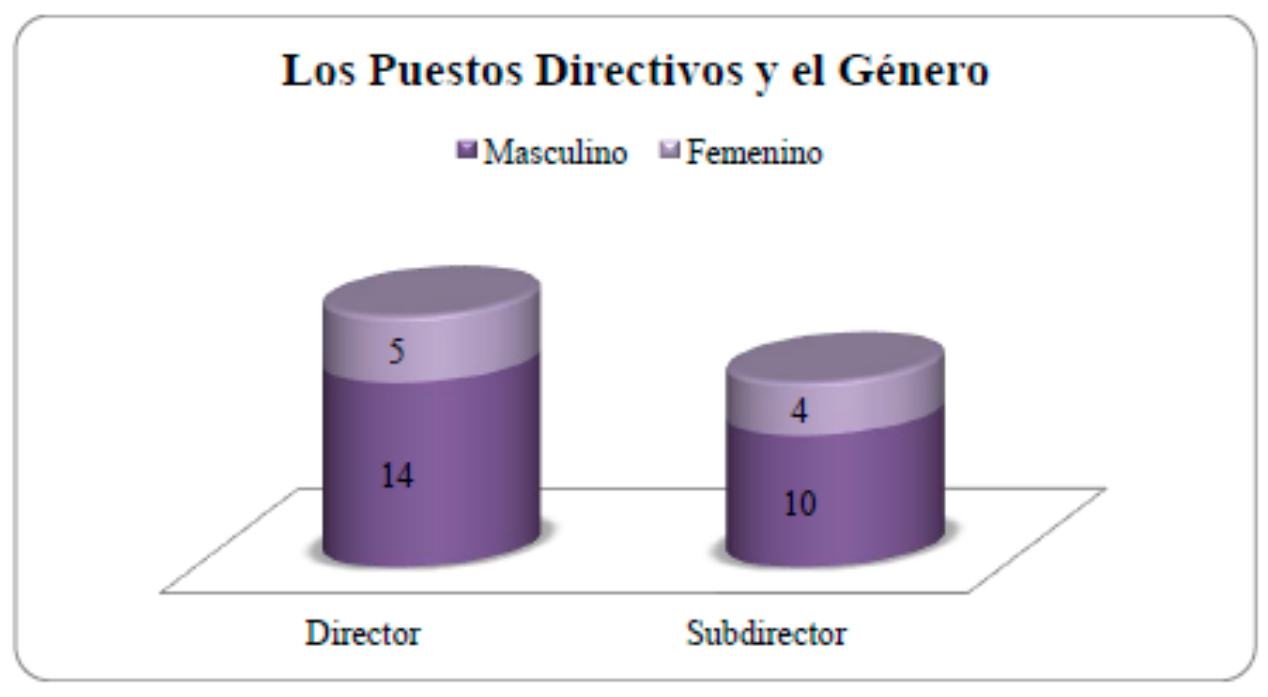

Figura 3. Los puestos directivos y el género.

De acuerdo con la información proporcionada por los directivos de 19 escuelas que forman parte de la población de estudio, del total de 476 docentes, 248 son mujeres; éstas representan el $52 \%$ de los docentes que laboran en las escuelas secundarias estatales de la ciudad de Chihuahua. Ello confirma la presencia mayoritaria del personal femenino en el ámbito de la docencia. Estas cifras son muy similares a "el indicador nacional de feminización en los servicios sociales y de la administración pública [pues] era de 104 mujeres por cada 100 hombres en 1995" (Izquierdo, 2001, 3)

Sin embargo, tal tendencia no se manifiesta en los puestos directivos, ya que en las escuelas que participan en el estudio la relación de mujeres y hombres docentes es de 1.08 a 1 respectivamente, cuando en los puestos directivos esta proporción disminuye; la relación en las subdirecciones es de 0.4 a 1 y en los puestos de dirección se mantiene a la baja, pues se presenta una relación de 0.3 a 1 . Ello no sólo confirma que en los puestos directivos continúa primando el ejercicio de las funciones por parte de personal que pertenece al género masculino, sino también que "a mayor nivel jerárquico, mayor presencia de hombres" (Izquierdo, 2001, 5)

De los 46 directivos que participaron durante la primera etapa de la investigación, 44 proporcionaron los datos del tiempo de servicio que poseen en el sistema educativo y de los años de experiencia que tienen en puestos directivos. A partir de esa información encontramos lo siguiente:

En promedio, el personal directivo de las escuelas secundarias estatales públicas de la ciudad de Chihuahua tiene 25.5 años de servicio. El directivo que ha permanecido menos tiempo, tiene en su haber 18 años de prestar un servicio educativo y el directivo con mayor trayectoria ha brindado durante 38 años sus servicios en el sistema educativo nacional.

El 66\% de los directivos tiene 25 años o más de servicio en el sistema educativo, mientras que el $13 \%$ de los directivos tiene menos de veinte años de servicio; es decir, seis directivos tienen menos de 20 años de servicio, y sólo uno ocupa el cargo de director. Ello representa una ventaja para el desempeño de la función directiva, ya que la mayor parte de los directivos se ha 
desempeñado en el sistema y por ende poseen experiencia en el terreno educativo.

El promedio de años de servicio en puestos directivos es de 6.9 años; sin embargo, hay profesores que no han cumplido ni un año como directivos; en este rubro también encontramos dos directores que llevan en el cargo más de 21 años. Esta información es posible ampliarla en la Figura 4.



Figura 4. Años de servicio en puestos directivos.

En lo referente al tiempo de servicio que tienen los directivos en la escuela, se encuentra que 7 de ellos acaban de integrarse en las instituciones en las que actualmente laboran. Al compararlo con la tercera etapa se observa una situación similar, esto se muestra en la figura 5.

\section{Tiempo de Servicio en Esta Escuela}

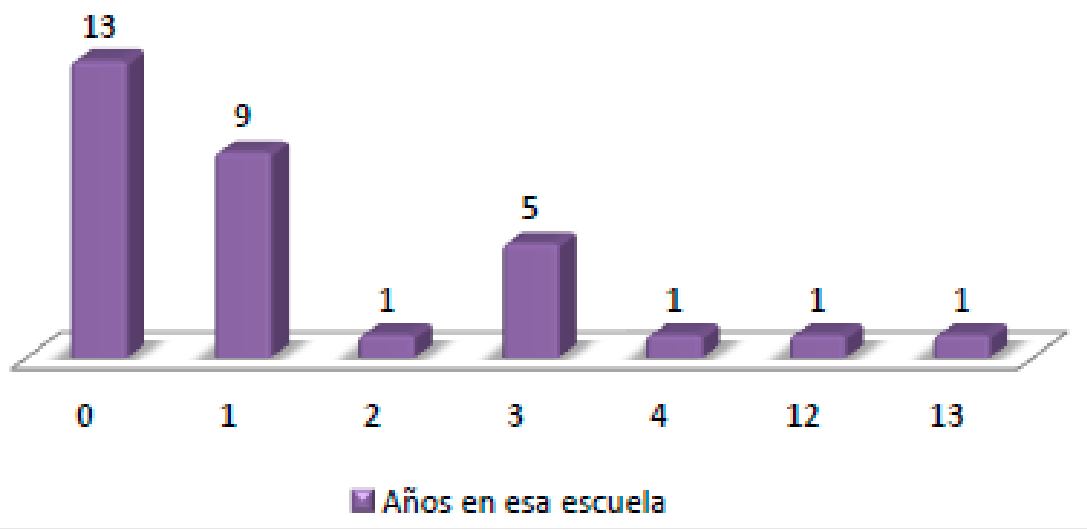

Figura 5. Tiempo de servicio en la escuela actual.

Sujetos de la Reforma: Condiciones institucionales para la implementación de la RS. 
De acuerdo con la información de la Figura 5, existen en la ciudad 2 directivos que cuentan con una gran trayectoria de servicio en puestos directivos en una misma escuela, uno de ellos tiene 12 años como directivo en un mismo centro escolar y otro lleva 13 años laborando en la misma escuela. Trece de los directivos prácticamente acaban de llegar a las escuelas en las que actualmente laboran, esta situación representa un reto para la implantación de la reforma, si consideramos por un lado la inestabilidad que se propicia al interior de los centros y por otro la falta de continuidad de los proyectos institucionales.

\section{Condiciones institucionales en las que se implanta la reforma}

A partir de la información recabada en las distintas etapas del estudio, ha sido posible describir las condiciones institucionales en las que se pone en marcha la reforma en las escuelas secundarias; con lo anterior nos referimos: al personal que conforma las escuelas; así como el equipamiento y la infraestructura que poseen las instituciones que participan en la investigación, sin embargo, por las limitantes que en la extensión del trabajo, sólo se incluye la primera sección.

\section{El personal que conforma las instituciones}

En las 26 escuelas del estudio laboran más de mil empleados que ejercen funciones docentes, administrativas, de asistencia educativa o de intendencia; todos son encabezados por los sujetos cuya perspectiva rescata esta investigación.

Los perfiles institucionales en los que se concreta formalmente el proceso de reforma son heterogéneos; en lo referente al número de empleados, encontramos centros conformados por 13 miembros, mientras que existen otros cuyo número asciende a 63 personas. El recorrido o rango del número de personal es de 50; eso confirma las disparidades respecto de la conformación de los colectivos escolares.

Pese al número de empleados que labora en las instituciones, no en todos los centros escolares existen ambas figuras administrativas (subdirector y director). El promedio de la cantidad de personal es 44 miembros, ese número pone de manifiesto la necesidad de contar con ambas figuras directivas para coordinar las funciones que debe ejercer cada integrante del personal, y no sólo reflejar estabilidad y una buena organización, sino encaminar a la institución de tal forma que con el quehacer cotidiano se vayan conformando las estructuras organizacionales para concretar y adaptarse a los cambios propuestos en la reforma.

\section{Referencias}

Elizondo, A. (2001) Dirección, liderazgo y gestión escolar. La nueva escuela, I y II. México: Paidós Mexicana.

Izquierdo, M. (2001). La feminización del magisterio en la base; en puestos directivos dominan varones. Consultado en agosto de 2011 en http://www.jornada.unam.mx/2001/ 11/05/arts_39/39_magisterio.htm

SEP (2004). Renovación pedagógica $y$ organizativa de las escuelas públicas de educación secundaria. Dirección General de Investigación Educativa de la Subsecretaría de Educación Básica y Normal México. Recuperado de: http://www.reformasecundaria.sep.g ob.mx/doc/gestionescolar/gestion.pdf SEP (2006). Plan de Estudios 2006. Educación Básica Secundaria. México: SEP. 